STUPP-92-132

November 10, 2018

\title{
Correlation Functions of $(2 k-1,2)$ Minimal Matter Coupled to 2D Quantum Gravity
}

\author{
SHUN-ICHI YAMAGUCHI \\ Physics Department, Faculty of Science \\ Saitama University \\ Urawa, Saitama 338, Japan
}

\begin{abstract}
We compute $N$-point correlation functions of non-unitary $(2 k-1,2)$ minimal matter coupled to 2D quantum gravity on a sphere using the continuum Liouville field approach. A gravitational dressing of the matter primary field with the minimum conformal weight is used as the cosmological operator. Our results are in agreement with the correlation functions of the one-matrix model at the $k$-th critical point.
\end{abstract}


Recent remarkable progress of $2 \mathrm{D}$ quantum gravity has been made by the matrix model approach. ${ }^{[1,2]}$ Matrix models provided us important and interesting understanding of non-perturbative aspects of 2D quantum gravity. Another approach called the Liouville approach ${ }^{[3,4]}$ uses a continuum field theory and also has been extensively studied. ${ }^{[5-12]}$ From the view point of the Liouville approach, it is important to compute correlation functions in order to study the non-trivial Liouville dynamics and in order to understand the precise connection with the matrix models. By using a free field approach and an analytic continuation procedure, $N$-point correlation functions for general $N$ without the screening charges in $c \leq 1$ conformal matters coupled to 2D gravity have been computed and analyzed in Refs. 10-12. They can be interpreted as scattering amplitudes of critical string theories in 2D target space with non-trivial background fields. In the case of $c<1$ minimal matters ${ }^{[13,14]}$ which require the screening charges, three-point functions have been computed. ${ }^{[6,11]}$ The results in unitary minimal matters were found to be consistent with the matrix model results.

In the Liouville approach, correlation functions of non-unitary minimal matters are not sufficiently understood so far. By comparing the gravitational dimensions ${ }^{[3,4]}$ of physical operators in the Liouville approach and the matrix model approach, one finds that a gravitational dressing of the matter primary field with the minimum conformal weight should be used as the cosmological operator. ${ }^{[5,9]}$ For unitary matters the cosmological operator is the ordinary one depending only on the Liouville field since the matter minimum weight primary field is the identity operator. On the other hand, for non-unitary minimal matters one must use a modified cosmological operator which depends on both of the matter and the Liouville fields.

In Ref. 15 three-point functions of non-unitary $(2 k-1,2)$ minimal matter ${ }^{[13]}$ were computed. However, two kinds of gravitational dressings of the matter identity operator were used as cosmological operators as in Ref. 8. It is not clear to us whether such a method is appropriate to non-unitary matters.

The purpose of this letter is to compute $N$-point functions of the non-unitary $(2 k-1,2)$ minimal matter coupled to $2 \mathrm{D}$ gravity on a sphere. They are obtained in the Liouville approach using the action with the modified cosmological operator. We use a similar technique as that used in Ref. 11 to compute $N$-point functions without screening charges. Our results are in agreement with the correlation functions of the one-matrix models at the $k$-th critical point, ${ }^{[2]}$ which are believed to represent the $(2 k-1,2)$ minimal matter coupled to $2 \mathrm{D}$ gravity.

Recently, we received a preprint by Govindarajan et al., ${ }^{[16]}$ in which $N$-point functions of the $(2 k-1,2)$ matter were obtained using different method from ours. They used algebraic properties of the BRST cohomology of the theory. Our results are also consistent with theirs.

We now consider the non-unitary $(2 k-1,2)$ minimal matter ${ }^{[13]}$ coupled to $2 \mathrm{D}$ quantum gravity. The matter conformal field theory has the central charge 


$$
c=1-12 \alpha_{0}^{2}
$$

with

$$
\alpha_{0}=-\frac{1}{2} \alpha_{+}+\frac{1}{2} \alpha_{-}, \quad \alpha_{+}=-\frac{2}{\sqrt{2 k-1}}, \quad \alpha_{-}=-\sqrt{2 k-1} .
$$

After conformal gauge fixing, the system is described by the matter field $X$ and the Liouville field $\phi$ with the action ${ }^{[4,14]}$

$$
\begin{aligned}
S[\hat{g}, \phi, X]= & \frac{1}{8 \pi} \int d^{2} z \sqrt{\hat{g}}\left(\hat{g}^{\alpha \beta} \partial_{\alpha} \phi \partial_{\beta} \phi-Q \hat{R} \phi\right)+\frac{\mu}{\pi} O_{\mathrm{m}} \\
& +\frac{1}{8 \pi} \int d^{2} z \sqrt{\hat{g}}\left(\hat{g}^{\alpha \beta} \partial_{\alpha} X \partial_{\beta} X+2 i \alpha_{0} \hat{R} X\right)+O_{+}+O_{-},
\end{aligned}
$$

where $\hat{R}$ is the scalar curvature on a sphere with a fixed reference metric $\hat{g}_{\alpha \beta}$ and $\mu$ is the cosmological constant. The operators $O_{ \pm}=\int d^{2} z \sqrt{\hat{g}} \mathrm{e}^{\mp i \alpha_{ \pm} X}$ are the matter screening charges and the operator $O_{\mathrm{m}}$ is the cosmological operator, which will be defined later. The parameter $Q$ is given by ${ }^{[4]}$

$$
Q=-\alpha_{+}-\alpha_{-}
$$

For each matter primary field $\Psi_{h}=\mathrm{e}^{i p X}$, which has a conformal weight $h=$ $\frac{1}{2} p^{2}-\alpha_{0} p$, there exists a physical operator ${ }^{[4,5]}$

$$
\begin{aligned}
& O_{p}^{( \pm)}=\int d^{2} z \sqrt{\hat{g}} \mathrm{e}^{\beta^{( \pm)} \phi+i p X} \\
& \beta^{( \pm)}(p)=-\frac{Q}{2}+\left|p-\alpha_{0}\right|=\left\{\begin{array}{lll}
p+\alpha_{+} & \cdots & \text { for } p \geq \alpha_{0} \\
-p+\alpha_{-} & \cdots & \text { for } p<\alpha_{0}
\end{array} .\right.
\end{aligned}
$$

For the $(2 k-1,2)$ matter the momentum $p$ of matter primary fields takes discrete values $^{[13,14]}$ parametrized by an integer $t$

$$
p=\left\{\begin{array}{l}
p_{t}=\frac{1}{2}(t-1) \alpha_{+} \geq \alpha_{0} \\
\bar{p}_{t}=\alpha_{-}-\frac{1}{2}(t+1) \alpha_{+}<\alpha_{0}
\end{array} \quad(1 \leq t \leq k-1)\right.
$$

Operators $O_{p_{t}}^{(+)}$and $O_{\bar{p}_{t}}^{(-)}$have the same matter conformal weight and represent the same operator of the minimal matter. The corresponding Liouville momenta are $\beta^{(+)}\left(p_{t}\right)=\beta^{(-)}\left(\bar{p}_{t}\right)=\frac{1}{2}(t+1) \alpha_{+}$. The cosmological operator $O_{\mathrm{m}}$ in Eq. (3) is $^{[5,9]}$ 


$$
O_{\mathrm{m}}=\int d^{2} z \sqrt{\hat{g}} \mathrm{e}^{\beta_{\mathrm{m}} \phi} \Psi_{h_{\mathrm{m}}}
$$

where $\Psi_{h_{\mathrm{m}}}\left(=\mathrm{e}^{i p_{\mathrm{m}} X}\right.$ or $\left.\mathrm{e}^{i \bar{p}_{\mathrm{m}} X}\right)$ is a matter primary field with the minimum conformal weight $h_{\mathrm{m}}$. It has a momentum $p_{\mathrm{m}}=p_{t=k-1}$ or $\bar{p}_{\mathrm{m}}=\bar{p}_{t=k-1}$ in Eq. (6) and $\beta_{\mathrm{m}}\left(=\beta_{\mathrm{m}}^{(+)}=\beta_{\mathrm{m}}^{(-)}\right)$in Eq. (5). The cosmological operator $O_{\mathrm{m}}$ is one of the physical operators in the theory.

We consider the $N$-point functions of the operators (5) with momenta (6) on a sphere. As in Refs. 6, 11, we first integrate the Liouville zero mode $\phi_{0}\left(\phi=\phi_{0}+\tilde{\phi}\right)$ and define $s_{\mathrm{m}}$ by

$$
\sum_{i=1}^{N} \beta_{i}^{(+)}+s_{\mathrm{m}} \beta_{\mathrm{m}}=-Q .
$$

Eq. (8) can be regarded as the conservation of the Liouville momentum. Next, we shall integrate the matter zero mode $X_{0}\left(X=X_{0}+\tilde{X}\right)$. Note that a factor $\left(\int d^{2} z \sqrt{\hat{g}} \mathrm{e}^{\beta_{\mathrm{m}}} \tilde{\phi} \Psi_{h_{\mathrm{m}}}\right)^{s_{\mathrm{m}}}$ resulting from the $\phi_{0}$ integration contributes to the matter zero mode integration. In order to simplify the matter momentum conservation and to symmetrize our final results under permutations of $\left(t_{1}, t_{2}, \cdots, t_{N}\right)$, without loosing generality we can use $s_{\mathrm{m}}-1 \mathrm{e}^{i p_{\mathrm{m}} X}$ 's and one $\mathrm{e}^{i \bar{p}_{\mathrm{m}} X}$ for $s_{\mathrm{m}} \Psi_{h_{\mathrm{m}}}$ 's. These two types of operators represent the same operator $\Psi_{h_{\mathrm{m}}}$. Then the matter momentum conservation can be satisfied by inserting $n$ screening charges $O_{+}$and no $O_{-}$

$$
\left[\sum_{i=1}^{N} p_{i}+\left(s_{\mathrm{m}}-1\right) p_{\mathrm{m}}+\bar{p}_{\mathrm{m}}\right]-n \alpha_{+}=-\alpha_{+}+\alpha_{-}
$$

Thus we obtain the result after the zero mode $X_{0}, \phi_{0}$ integrations

$$
\begin{aligned}
& \left\langle O_{1}^{(+)} \cdots O_{N}^{(+)}\right\rangle \\
& =2 \pi \delta\left(\sum_{i=1}^{N} p_{i}+\left(s_{\mathrm{m}}-1\right) p_{\mathrm{m}}+\bar{p}_{\mathrm{m}}+(1-n) \alpha_{+}-\alpha_{-}\right) \frac{\Gamma\left(-s_{\mathrm{m}}\right)}{\left|\beta_{\mathrm{m}}\right|}\left(\frac{\mu}{\pi}\right)^{s_{\mathrm{m}}} \\
& \quad \times\left\langle\tilde{O}_{1}^{(+)}(0) \tilde{O}_{2}^{(+)}(\infty) \tilde{O}_{3}^{(+)}(1) \tilde{O}_{4}^{(+)} \cdots \tilde{O}_{N}^{(+)}\left[\left(\tilde{O}_{\mathrm{m}}^{(+)}\right)^{s_{\mathrm{m}}-1} \tilde{O}_{\mathrm{m}}^{(-)}\right] \frac{1}{n !}\left(\tilde{O}_{+}\right)^{n}\right\rangle,
\end{aligned}
$$

where we have used shorthand notations $\tilde{O}_{i}(z)=\mathrm{e}^{\beta_{i} \tilde{\phi}+i p_{i} \tilde{X}}, \tilde{O}_{i}=\int d^{2} z \sqrt{\hat{g}} \tilde{O}_{i}(z)$ etc. and fixed $S L(2, \mathbf{C})$ gauge symmetry by setting $z_{1}=0, z_{2}=\infty, z_{3}=1$. The non-zero mode $\tilde{\phi}, \tilde{X}$ expectation value on the right hand side of Eq. (10) is defined by using the free field action. 
To evaluate the non-zero mode expectation value in Eq. (10), let us consider more general cases

$$
\left\langle\tilde{O}_{1}^{(+)}(0) \tilde{O}_{2}^{(+)}(\infty) \tilde{O}_{3}^{(+)}(1) \tilde{O}_{4}^{(+)} \cdots \tilde{O}_{M-1}^{(+)} \tilde{O}_{M}^{(-)}\left(\tilde{O}_{c}\right)^{s} \frac{1}{n !}\left(\tilde{O}_{+}\right)^{n}\right\rangle
$$

where $O_{c}=\int d^{2} z \sqrt{\hat{g}} \mathrm{e}^{\alpha_{+} \phi}$ is a physical operator $O_{p=0}^{(+)}$in Eq. (5). The matter momentum conservation and the definition of $s$ are

$$
\sum_{i=1}^{M} p_{i}-n \alpha_{+}=-\alpha_{+}+\alpha_{-}, \quad \sum_{i=1}^{M} \beta_{i}+s \alpha_{+}=-Q .
$$

The expectation value in Eq. (10) corresponds to $s=0, M=N+s_{\mathrm{m}}$ and $p_{i}=p_{\mathrm{m}}$ $\left(i=N+1, \cdots, N+s_{\mathrm{m}}-1\right), p_{i=N+s_{\mathrm{m}}}=\bar{p}_{\mathrm{m}}$. Eq. (11) for $M=3$ was evaluated in Ref. 11 (see also Ref. 6) using the techniques of analytic continuations in the momenta and the matter central charge. The non-zero mode integration is performed by tuning the momenta $p_{1}, p_{2}, p_{3}$ and the matter central charge $c$ so that $s$ and $n$ are non-negative integers. The expectation value with desired values of the momenta and the central charge is then obtained by an analytic continuation. In this way the three-point function was obtained as ${ }^{[1]]}$

$$
\begin{aligned}
& \left\langle\tilde{O}_{1}^{(+)}(0) \tilde{O}_{2}^{(+)}(\infty) \tilde{O}_{3}^{(-)}(1)\left(\tilde{O}_{c}\right)^{s} \frac{1}{n !}\left(\tilde{O}_{+}\right)^{n}\right\rangle \\
& =\pi^{s+n} \frac{\Gamma(s+1)}{[\Gamma(s+n+1)]^{2}}[\Delta(-\rho)]^{s}[\Delta(\rho)]^{n} \prod_{i=1}^{2} \Delta\left(m_{i}\right),
\end{aligned}
$$

where

$$
\rho=-\frac{1}{2} \alpha_{+}^{2}, \quad m_{i}=m\left(p_{i}\right)=\frac{1}{2} \beta_{i}^{2}-\frac{1}{2} p_{i}^{2}, \quad \Delta(x)=\frac{\Gamma(x)}{\Gamma(1-x)} .
$$

In Ref. $11 M$-point functions (11) for general $M$ without screening charge $n=0$ were also obtained. It was pointed out that the matter screening charges should not be treated on the equal footing as physical operators (5). For example, using the analytic continuation procedure, the expectation value (11) for $M=3$ with $n$ screening charges can not be obtained from that of $M=3+n$ with no screening charge.

We shall now obtain the four-point function. We closely follow the procedure of Ref. 11 to derive four-point functions from three-point functions when there is no screening charge. From the kinematical constraints (12) $m_{i}$ satisfy 


$$
\sum_{i=1}^{3} m_{i}=(s-n) \rho+1, \quad m_{4}=-s-n-1,
$$

and we can take $m_{1}$ and $m_{3}$ as independent variables. Eq. (11) for $M=4$ and $s, n \in \mathbf{Z}_{+}$becomes

$$
\begin{aligned}
& \left\langle\tilde{O}_{1}^{(+)}(0) \tilde{O}_{2}^{(+)}(\infty) \tilde{O}_{3}^{(+)}(1) \tilde{O}_{4}^{(-)}\left(\tilde{O}_{c}\right)^{s} \frac{1}{n !}\left(\tilde{O}_{+}\right)^{n}\right\rangle \\
& =\frac{1}{n !} \int d^{4} z_{4}\left|z_{4}\right|^{2 a_{1}}\left|1-z_{4}\right|^{2 a_{3}} \\
& \quad \times \int \prod_{i=1}^{s}\left[d^{2} w_{i}\left|w_{i}\right|^{2 b_{1}^{+}}\left|1-w_{i}\right|^{2 b_{3}^{+}}\left|z_{4}-w_{i}\right|^{2 c^{-}}\right] \prod_{1 \leq i<j \leq s}\left|w_{i}-w_{j}\right|^{4 \rho} \\
& \quad \times \int \prod_{i=1}^{n}\left[d^{2} u_{i}\left|u_{i}\right|^{2 b_{1}^{-}}\left|1-u_{i}\right|^{2 b_{3}^{-}}\left|z_{4}-u_{i}\right|^{2 c^{+}}\right] \prod_{1 \leq i<j \leq n}\left|u_{i}-u_{j}\right|^{-4 \rho} \\
& \quad \equiv F\left(m_{1}, m_{3} ; \rho, s, n\right)
\end{aligned}
$$

where

$$
a_{i}=(s+n+1) m_{i}-1, \quad b_{i}^{ \pm}=-m_{i} \pm \rho, \quad c^{ \pm}= \pm(s+n+1) \rho-1 .
$$

We first consider $F$ as a function of $m_{1}$ and discuss its analyticity. From the integral representations (16), one can see that poles in $m_{1}$ arise when some of the integration variables $z_{4}, w_{i}, u_{i}$ approach to the origin or infinity. Let us first examine the poles arising from integration regions near the origin. The positions of these poles are independent of $m_{3}$. Therefore we can find them by setting $p_{3}=0$, which reduces (16) to the three point function (13) with $s \rightarrow s+1$ for the operators $\tilde{O}_{1}, \tilde{O}_{2}$ and $\tilde{O}_{4}$. Thus, from Eq. (13) we find that the poles arise only when $\tilde{O}_{4}$ approaches to $\tilde{O}_{1}\left(z_{4} \rightarrow 0\right)$ and their positions are $m_{1}=0,-1,-2, \cdots$. Next we shall examine the poles arising from integration regions near infinity. They can be naturally understood in terms of $m_{2}=-m_{1}-m_{3}+(s-n) \rho+1$. By changing the integration variables $z\left(=z_{4}, w_{i}, u_{i}\right) \rightarrow 1 / z$, we find that these poles arise when $\tilde{O}_{4}$ approaches to $\tilde{O}_{2}$ and their positions are $m_{2}=0,-1,-2 \cdots$. By similar arguments we find that poles in $m_{3}$ arise at $m_{3}=0,-1,-2 \cdots$. There is no other singularity in Eq. (16).

Let us consider the quantity

$$
f\left(m_{1}, m_{3} ; \rho, s, n\right)=\frac{F\left(m_{1}, m_{3} ; \rho, s, n\right)}{\Delta\left(m_{1}\right) \Delta\left(m_{2}\right) \Delta\left(m_{3}\right)} .
$$

We can show that $f\left(m_{1}, m_{3} ; \rho, s, n\right)$ is independent of $m_{1}$ and $m_{3}$ as follows. ${ }^{[11]}$ Changing the integration variables $z^{\prime}\left(=z_{4}^{\prime}, w_{i}^{\prime}, u_{i}^{\prime}\right)=m_{1} \ln z$ in Eq. (16), we find 
that $F \sim\left|m_{1}\right|^{-2 m_{3}+2(s-n) \rho}$ for large $\left|m_{1}\right|$ and therefore $f$ is a constant for $\left|m_{1}\right| \rightarrow$ $\infty$. The singularities of $F$ in $m_{1}$ are canceled by those of $\Delta\left(m_{i}\right)$. Furthermore, it can be shown in the same way as in Ref. 11 that $F$ vanishes at $m_{1}=1,2,3, \cdots$, which are zeros of the denominator in Eq. (18). Thus we find that $f$ is an analytic function on the whole complex $m_{1}$-plane including a point at infinity. Therefore it must be a constant independent of $m_{1}$. Similarly $f$ is shown to be independent of $m_{3}$. The explicit form of $f(\rho, s, n)$ is obtained as follows. If we put one of the momenta to zero in Eq. (16), it is reduced to a three-point function with $s+1 \tilde{O}_{c}$ 's. By comparing with Eq. (13), we obtain

$$
f(\rho, s, n)=\pi^{s+n+1} \frac{\Gamma(s+2)}{[\Gamma(s+n+2)]^{2}}[\Delta(-\rho)]^{s}[\Delta(\rho)]^{n} .
$$

From Eqs. (18) and (19) we obtain

$$
\left\langle\tilde{O}_{1}^{(+)}(0) \tilde{O}_{2}^{(+)}(\infty) \tilde{O}_{3}^{(+)}(1) \tilde{O}_{4}^{(-)}\left(\tilde{O}_{c}\right)^{s} \frac{1}{n !}\left(\tilde{O}_{+}\right)^{n}\right\rangle=f(\rho, s, n) \prod_{i=1}^{3} \Delta\left(m_{i}\right)
$$

We can easily generalize above arguments to $M$-point $(M \geq 5)$ functions. The variables $m_{i}$ satisfy

$$
\sum_{i=1}^{M-1} m_{i}=(s-n) \rho+1, \quad m_{M}=-s-n-M+3 .
$$

The analyticity in $m_{i}$ and comparison with lower point functions give the result ${ }^{\star}$

$$
\begin{aligned}
& \left\langle\tilde{O}_{1}^{(+)}(0) \tilde{O}_{2}^{(+)}(\infty) \tilde{O}_{3}^{(+)}(1) \tilde{O}_{4}^{(+)} \ldots \tilde{O}_{M-1}^{(+)} \tilde{O}_{M}^{(-)}\left(\tilde{O}_{c}\right)^{s} \frac{1}{n !}\left(\tilde{O}_{+}\right)^{n}\right\rangle \\
& \quad=\pi^{s+n+M-3} \frac{\Gamma(s+M-2)}{[\Gamma(s+n+M-2)]^{2}}[\Delta(-\rho)]^{s}[\Delta(\rho)]^{n} \prod_{i=1}^{M-1} \Delta\left(m_{i}\right) .
\end{aligned}
$$

Let us now obtain the non-zero mode expectation values in Eq. (10) using the above results. By setting $s=0, M=N+s_{\mathrm{m}}$ and $p_{i}=p_{\mathrm{m}}\left(i=N+1, \cdots, N+s_{\mathrm{m}}-1\right)$, $p_{i=N+s_{\mathrm{m}}}=\bar{p}_{\mathrm{m}}$ in Eq. (22), we obtain

* This result is also useful for unitary minimal matters coupled to $2 \mathrm{D}$ gravity, in which the cosmological operator is $O_{c}$. 


$$
\begin{gathered}
\left\langle\tilde{O}_{1}^{(+)}(0) \tilde{O}_{2}^{(+)}(\infty) \tilde{O}_{3}^{(+)}(1) \tilde{O}_{4}^{(+)} \cdots \tilde{O}_{N}^{(+)}\left[\left(\tilde{O}_{\mathrm{m}}^{(+)}\right)^{s_{\mathrm{m}}-1} \tilde{O}_{\mathrm{m}}^{(-)}\right] \frac{1}{n !}\left(\tilde{O}_{+}\right)^{n}\right\rangle \\
=\pi^{s_{\mathrm{m}}+n+N-3} \frac{\Gamma\left(s_{\mathrm{m}}+N-2\right)}{\left[\Gamma\left(s_{\mathrm{m}}+n+N-2\right)\right]^{2}}[\Delta(\rho)]^{n}\left[\Delta\left(m_{\mathrm{m}}\right)\right]^{s_{\mathrm{m}}-1} \prod_{i=1}^{N} \Delta\left(m_{i}\right),
\end{gathered}
$$

where $m_{\mathrm{m}}=\frac{1}{2} \beta_{\mathrm{m}}^{2}-\frac{1}{2} p_{\mathrm{m}}^{2}$. The result (23) obtained for non-negative integers $s_{\mathrm{m}}$ is now analytically continued to a desired value of $s_{\mathrm{m}}$. Therefore, by Eq. (23) we find that the $N$-point function (10) becomes

$$
\begin{aligned}
\left\langle O_{1}^{(+)} \cdots O_{N}^{(+)}\right\rangle= & 2 \pi \delta\left(\sum_{i=1}^{N} p_{i}+\left(s_{\mathrm{m}}-1\right) p_{\mathrm{m}}+\bar{p}_{\mathrm{m}}+(1-n) \alpha_{+}-\alpha_{-}\right) \\
& \times \frac{\mu^{s_{\mathrm{m}}}}{\left|\beta_{\mathrm{m}}\right|} \frac{\Gamma\left(s_{\mathrm{m}}+N-2\right)}{\Gamma\left(s_{\mathrm{m}}+1\right)}(-\pi)^{n+N-3}[\Delta(\rho)]^{n} \\
& \times\left[\Delta\left(m_{\mathrm{m}}\right)\right]^{s_{\mathrm{m}}-1} \Delta\left(-s_{\mathrm{m}}-n-N+3\right) \prod_{i=1}^{N} \Delta\left(m_{i}\right) .
\end{aligned}
$$

From Eqs. (8) and (9) we obtain

$$
s_{\mathrm{m}}=\frac{1}{k}\left(2 k+1-N-\sum_{i=1}^{N} t_{i}\right), \quad n=-s_{\mathrm{m}}-N+\frac{5}{2},
$$

where $t_{i}$ is parameters for momenta $p_{i}$ in Eq. (6). The parameters defined in Eq. (14) take the values

$$
\rho=-\frac{2}{2 k-1}, \quad m_{i}=\frac{2 t_{i}}{2 k-1}, \quad m_{\mathrm{m}}=\frac{2 k-2}{2 k-1} .
$$

Substituting Eqs. (25) and (26) into Eq. (24) we obtain the final result of the $N$-point function.

Let us compare this result with that of the matrix model approach. The normalizations of the partition function and the physical operators may be different in two approaches. We rescale the partition function and renormalize the operators and the cosmological constant by finite real factors as 


$$
\begin{aligned}
Z & \rightarrow-[\pi(1-2 k)]^{-\frac{1}{2}}\left[\Delta\left(\frac{2 k+1}{2 k-1}\right)\right]^{\frac{5}{2}} \Delta\left(\frac{2 k-2}{2 k-1}\right) Z \\
O_{p_{t_{i}}}^{(+)} & =\Delta\left(\frac{2 k+1}{2 k-1}\right) \Delta\left(\frac{2 t_{i}}{2 k-1}\right) O_{l_{i}} \quad\left(l_{i} \equiv k-1-t_{i}\right) \\
\mu & =-\pi \Delta\left(-\frac{2}{2 k-1}\right) \Delta\left(\frac{1}{2 k-1}\right) \mu_{r}
\end{aligned}
$$

and obtain

$$
\left\langle O_{l_{1}} \cdots O_{l_{N}}\right\rangle=-\frac{1}{k} \frac{\Gamma\left[\left(\sum_{i=1}^{N} l_{i}+1\right) / k\right]}{\Gamma\left[\left(\sum_{i=1}^{N} l_{i}+1-(N-3) k\right) / k\right]} \mu_{r}\left[\sum_{i=1}^{N} l_{i}+1-(N-2) k\right] / k .
$$

The integer parameters $l_{i}$ take values $0,1, \cdots, k-2$. This result is in agreement with the correlation functions of the one-matrix model at the $k$-th critical point. ${ }^{[2] \star}$

Acknowledgements: The author would like to thank Y. Tanii for many valuable discussions and very careful reading of this manuscript. He is also grateful to members of the theory group at Saitama University for useful comments and encouragements.

$\star$ There are misprints in the formula for the correlation functions (Eq. (4.19)) given in the first paper of Ref. 2. 


\section{REFERENCES}

1. E. Brézin and V.A. Kazakov, Phys. Lett. 236B (1990) 144; M. Douglas and A. Shenker, Nucl. Phys. B335 (1990) 635; D.J. Gross and A.A. Migdal, Phys. Rev. Lett. 64 (1990) 127.

2. D.J. Gross and A.A. Migdal, Nucl. Phys. B340 (1990) 333; T. Banks, M. Douglas, N. Seiberg and S. Shenker, Phys. Lett. 238B (1990) 279.

3. V.G. Knizhnik, A.M. Polyakov and A.B. Zamolodchikov, Mod. Phys. Lett. A3 (1988) 819.

4. F. David, Mod. Phys. Lett. A3 (1988) 1651; J. Distler and H. Kawai, Nucl. Phys. B321 (1989) 509.

5. N. Seiberg, Prog. Theor. Phys. Suppl. 102 (1990) 319; J. Polchinski, in Strings '90, eds. R. Arnowitt et al. (World Scientific, Singapore, 1991).

6. M. Goulian and M. Li, Phys. Rev. Lett. 66 (1991) 2051; Y. Kitazawa, Phys. Lett. 265B (1991) 262; Int. J. Mod. Phys. A7 (1992) 3403; K. Aoki and E. D'Hoker, Mod. Phys. Lett. A7 (1992) 235.

7. Y. Tanii and S. Yamaguchi, Mod. Phys. Lett. A6 (1991) 2271.

8. V.S. Dotsenko, Mod. Phys. Lett. A6 (1991) 3601.

9. L. Alvarez-Gaumé, J.L.F. Barbón and C. Gómez, Nucl. Phys. B368 (1992) 57.

10. A.M. Polyakov, Mod. Phys. Lett. A6 (1991) 635; D. Minic and Z. Yang, Phys. Lett. 274B (1992) 27; N. Sakai and Y. Tanii, Phys. Lett. 276B (1992) 41; to appear in Prog. Theor. Phys. Suppl.

11. P. Di Francesco and D. Kutasov, Phys. Lett. 261B (1991) 385; Nucl. Phys. B375 (1992) 119.

12. M. Bershadsky and D. Kutasov, Phys. Lett. 274B (1992) 331; Y. Tanii and S. Yamaguchi, Mod. Phys. Lett. A7 (1992) 521; Saitama preprint STUPP92-128 (March 1992).

13. A.A. Belavin, A.M. Polyakov and A.B. Zamolodchikov, Nucl. Phys. B241 (1984) 333.

14. V.S. Dotsenko and V.A. Fateev, Nucl. Phys. B240 (1984) 312; B251 (1985) 691; V.S. Dotsenko, Advanced Studies in Pure Mathematics 16 (1988) 123.

15. D. Ghoshal and S. Mahapatra, Tata preprint TIFR-TH-91-58 (December, 1991).

16. S. Govindarajan, T. Jayaraman and V. John, Madras preprint, IMSc-92/35 (August, 1992). 